\title{
Tratamiento de la clase II división 2 con la técnica Bioprogresiva de Ricketts
}

Treatment of Class II division 2 with Ricketts Bioprogressive technique

\begin{abstract}
Resumen
El manejo clínico de la Maloclusión de Clase II 2da División depende de un buen diagnóstico y planeamiento del caso. La mayoría de estos pacientes deberían ser tratados sin extracciones, dadas las características faciales que presentan y la posibilidad de crear espacios mediante métodos de distalización de molares y cambio de inclinación e intrusión de los incisivos. El presente caso clínico nos muestra como podemos hacer uso del diagnóstico basado en la información que nos proporciona la cefalometría y proponer un tratamiento basado en el Objetivo Visual de Tratamiento(OVT) además de visualizar la utilidad de la biomecánica de la Técnica Bioprogresiva de Ricketts en los pacientes de Clase II División 2.
\end{abstract}

\section{Abstract}

The clinical handling of malocclusions Class II Division 2 depends on a good diagnosis and planning of the case. Most of these patients should be treated without teeth extractions, regarding that facial characteristics they present and the real possibility to create spaces by molars distalization methods and changing the incisives inclinations and intrusion. The present clinical case shows us how we can make use of diagnosis based on the information that the cephalometry provides and propose a treatment based on the Visual Treatment Objective (VTO) as well as to visualize the utility of biomechanics of Ricketts's Bioprogressive technique on patients Class II Division 2.

\section{Introducción}

La maloclusión de Clase II división 2 presenta características muy especiales en donde lo mas relevante es la reproposición de los incisivos superiores e inferiores y una sobremordida exagerada. Tendencia de crecimiento braquifacial y componente neuromuscular acentuado. ${ }^{1}$

Los datos epidemiológicos demuestran que esta maloclusión varia entre un 2,6 $\%$ a un $8,2 \%$ para las poblaciones de origen europeo. ${ }^{2,3}$ Van der Linden4 las clasifica en tres tipos A, B y C. Existe un gran rango de variabilidad en la severidad de estas maloclusiones y el grado de dificultad del tratamiento esta en directa relación con la expresión de los parámetros arriba señalados. La etiopatogenia aun no es del todo conocida, aunque se sabe que existe un fuerte componente hereditario en donde el rasgo común en los miembros de una misma familia es la retroinclinación de los incisivos. Otras hipótesis tienden a explicar el importante papel que tiene la musculatura en la determinación de la inclinación de los incisivos tras la erupción. .,6 Van $^{5}$ der Linden ${ }^{4}$ destaca la importancia de la posición alta de la línea labial que condiciona la reproposición de los incisivos durante la erupción.

Cefalométricamente presentan una relación esquelética de Clase II (en donde el problema es la reproposición de la mandíbula) o en el mejor de los casos Clase I .Los planos palatinos y mandibulares casi paralelos, disminución del tercio inferior de la cara, deflexión craneana y eje facial con tendencia braquifacial y casi siempre con un ángulo mandibular bajo. El perfil total se muestra convexo, pero el perfil del tercio inferior tiende a ser cóncavo. $\mathrm{El}$ arco mandibular generalmente con valores de normal a alto, lo cual configura un neuromuscular sumamente fuerte. Los incisivos superiores se encuentran retruidos e inclinados a palatino y los inferiores retruidos e inclinados a lingual. Esta verticalización de los incisivos superiores coloca al punto A hacia delante, dándonos una

\section{Luis Fernando Pérez Vargas ${ }^{1}$, Ana María Díaz Soriano ${ }^{1}$}

Departamento Académico de Estomatología Biosocial de la Facultad de Odontología de la UNMSM

Correspondencia:

Mg. Esp. Luis Fernando Pérez Vargas. Facultad Odontología, UNMSM

Av. Germán Amézaga s/n, Lima, 1 Perú. e-mail: lperezv@unmsm.edu.pe

Palabras clave: Maloclusión Clase II división 2, Bioprogresiva, Distalización de molares.

Key words: Class II Division 2. malocclusion, Bioprogressive. Molar distalization.

falsa lectura de una protrusión de la maxila; de igual manera la mandíbula retroposicionada con una hipertrofia de los músculos mentalis condiciona el desarrollo de un mentón prominente y por lo tanto una lectura errónea de una mandíbula de tamaño grande. La arca inferior dada estas condiciones puede observarse claramente apiñada y la arcada superior puede que falte espacio para la erupción del canino. Esto puede llevar al clínico inexperto a tomar la decisión de extraer piezas para eliminar la discrepancia arco diente. La decisión de extraer o no extraer, deberá ser tomada luego de una cuidadosa evaluación facial del paciente. ${ }^{78}$ Frecuentemente presentan la nariz y el mentón prominente, además de un tercio inferior de la cara cóncavo y disminuido. Las extracciones podrían no ser la mejor alternativa para estos pacientes ya que tienden a empeorar las condiciones faciales. Demás es sabido que poseen un neuromuscular sumamente fuerte y son de los pacientes que tienden a recidivar con mucha frecuencia. 
Los criterios terapéuticos que señalan los autores, están encaminados a la corrección de la sobremordida para liberar la mandíbula, reducción del ángulo ínter incisivo para consolidar la corrección vertical y aumento de la longitud de la arcada por medios conservadores tales como expansión de la arcada, distalización de molares superiores y proinclinación de los incisivos.

\section{Caso Clínico}

\section{Sumario de diagnóstico:}

- Paciente de sexo femenino de 12 años, en crecimiento, que presenta una Maloclusión de Clase II división 2 tipo B y una relación esquelética de Clase I.

- Incisivos superiores retruidos e inclinados a palatino e inferiores retruidos.

- Presenta una maxila de tamaño normal y en buena posición.

- Mandíbula grande y mentón protruido.

- Presenta una dirección de crecimiento mandibular proporcional

- Presenta un fuerte neuromuscular

- Discrepancia arco diente superior $=-4.06 \mathrm{~mm}$

- Discrepancia arco diente inferior $=$ $-4.3 \mathrm{~mm}$

- Discrepancia de tamaño Bolton Exceso inferior (6 pzas. anteroinferiores) $=2.05 \mathrm{~mm}$

- Línea media superior centrada

- Línea media inferior desviada 2mm a la izquierda

- Over bite alterado

- AFAI disminuido

- AFT disminuido

- Perfil convexo

\section{Objetivos de tratamiento}

1. Corregir la Maloclusión y mantener la relación esquelética.

2. Mejorar la posición e inclinación de los incisivos.

3. Eliminar la discrepancia arco diente superior e inferior.

4. Eliminar la discrepancia de Bolton.

5. Mejorar línea media inferior.

6. Mejorar la altura facial ántero inferior.

7. Mejorar el perfil.

8. Mejorar la guía incisiva y la guía canina.

\section{Plan de tratamiento}

El tratamiento consistió en la distalización de los molares superiores con aparato pendular de Hilgers, arcos utilitarios de vestibularización e intru- sión de incisivos con arcos seccionales de alineamiento y nivelación. Arcos de retrusión de incisivos con elásticos intermaxilares de Clase II. Arcos y elásticos de finalización.

\section{Procedimiento clínico}

La cefalometría nos brinda valiosa información antes de efectuar los procedimientos ortodóncicos. El paciente presentaba una posición mesial de las molares superiores evaluado por la distancia Pt vertical a distal de la molar superior la cual era de $18 \mathrm{~mm}$, siendo el valor normal según Ricketts la edad más $3 \mathrm{~mm}$, lo cual quería decir que la molar debería estar situada a $15 \mathrm{~mm}$ (edad: 12 más $3=15 \mathrm{~mm}$ ) dándonos la posibilidad de distalizar aproximadamente $3 \mathrm{~mm}$. Además era necesario que se abriera el eje para mejorar la altura facial antero inferior (ENA- Xi pm $=39$ ). Por estas razones se escogió el aparato pendular de Hilgers $^{9,10}$, que si bien es cierto, produce extrusión de las molares el paciente también presentaba un fuerte neuromuscular (arco mandibular $=43^{\circ}$ ) que le permitiría contrarrestar dichos efectos adversos (ver cefalometría inicial ). Por otro lado la predicción de crecimiento sin tratamiento nos indicaba que la molar crecería hacia delante y hacia abajo (ver predicción de crecimiento) es decir el crecimiento no mejoraría la relación molar de Clase II sino que por el contrario la empeoraría. Por otro lado se procedió a cambiar la inclinación de los incisivos superiores con una serie de arcos utilitarios Engiloy azul 0,016"x 0.016" lo que nos permitió mayor perímetro en el arco. La intrusión posterior a la vestibularización permitió mejorar el over bite y over jet.

En el arco inferior se colocaron arcos continuos de alineamiento y nivelación NitiCu 0.016", redondo acero $0.016^{\prime \prime}$ y acero rectangular $0.016^{\prime \prime} \mathrm{x}$ $0.022^{\prime \prime}$ con el propósito de promover el alineamiento de los dientes pero también el crecimiento y suave extrusión efecto que ayudaría a abrir el eje facial y mejorar el tercio inferior de la cara previstos en el VTO (ver VTO).

Lograda la distalización de las molares, se procedió a la distalización de los premolares y caninos con arcos seccionales y elásticos intermaxilares de Clase II, hasta lograr una relación canina de Clase I. Luego se colocó un arco delta para retruir y consolidar los incisivos con los dientes posteriores. Se agregó un torque adicional al arco para mantener la inclinación de los incisivos. Se realizó un suave desgaste inter- dental en los seis anteriores inferiores con lijas para eliminar la discrepancia de Bolton. Para la ínter digitación final se utilizaron elásticos intermaxilares de $3 / 4$. Para la contención se utilizaron arco de Hawley continuo superior y arco lingual adherido 3-3.

\section{Discusión}

La evaluación y discusión de los resultados la haremos en función de los objetivos propuestos.

La publicación del presente caso clíni$\mathrm{co}$, se realiza con el conocimiento informado de los padres de la paciente, expresado por escrito.

1. Corregir la Maloclusión y mantener la relación esquelética. Los resultados clínicos nos permiten observar que se logro la corrección de la maloclusión. La evaluación cefalométrica de la distalización de las molares superiores nos permiten concluir que la corrección de la Clase II es, en gran parte por la inhibición del crecimiento natural de la molar hacia abajo y adelante y ligera distalización real de la molar .En cuanto a la relación esquelética esta se mantuvo en clase I tal como se puede verificar en la cefalometría final (plano facial- $A=4 \mathrm{~mm}$ ). A pesar que el eje facial se abrió y la mandíbula rotó hacia abajo y hacia atrás, este efecto fue compensado por una inhibición del crecimiento anteroposterior de la maxila. (ver superposiciones finales).

2. Mejorar la posición e inclinación de losincisivos. Los incisivos superiores mejoraron sustancialmente con la terapia la posición e inclinación. Esto se debe en gran parte al uso de arcos utilitarios que permitieron un control en el torque. El incisivo inferior se protruyó pero mantuvo su inclinación.

3. Eliminar la discrepancia arco diente superior e inferior. Esto se logró en gran parte por la distalización molar, cambio de inclinación de incisivos y efecto expansivo de los arcos utilitarios. En el arco inferior el desgaste interproximal de los seis anteriores y el efecto expansivo del arco continuo permitió la eliminación de la discrepancia.

4. Eliminar la discrepancia de Bolton. Se eliminó con desgaste interproximales de los seis anteriores.

5. Mejorar línea media inferior. Se mejoró pero no llegó a corregirse. 
Figura 1.Fotografías extraorales e intraorales que muestran la maloclusión de Clase II División 2.
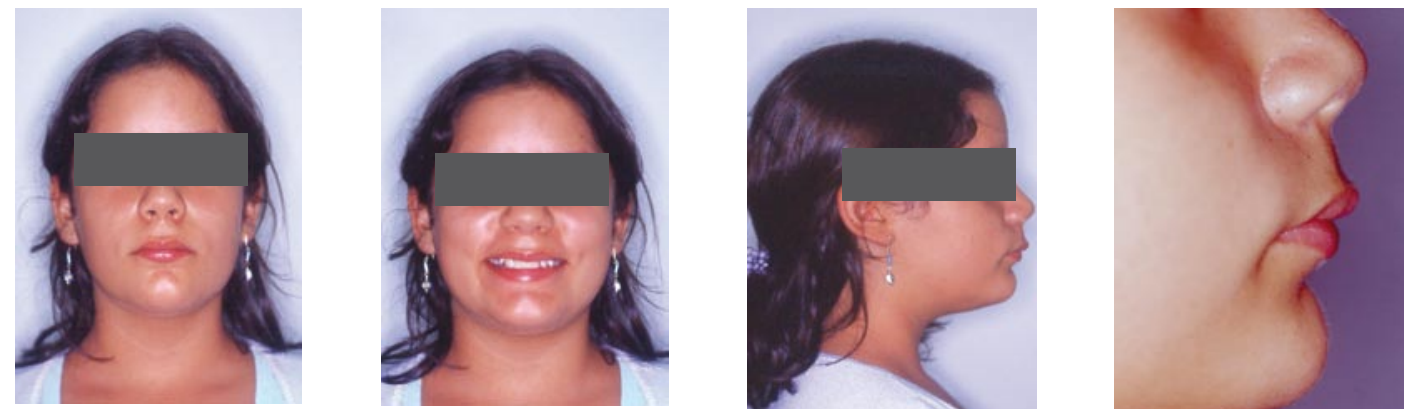
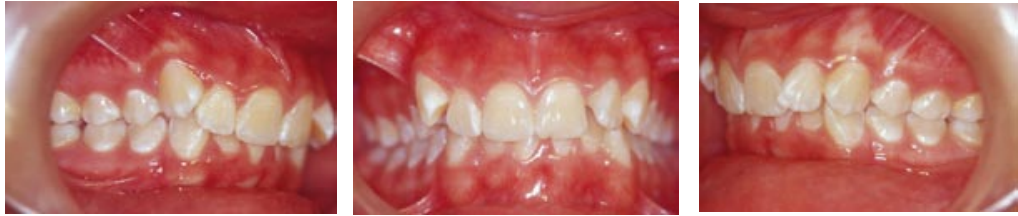

Figura 2. Radiografía cefalométrica y panorámica
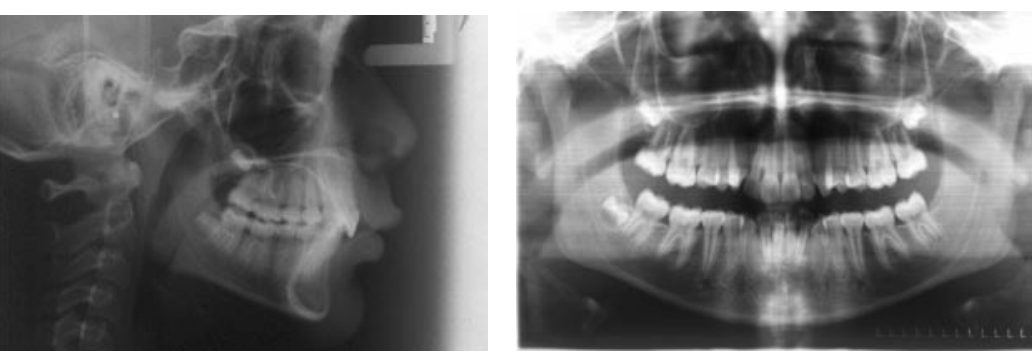

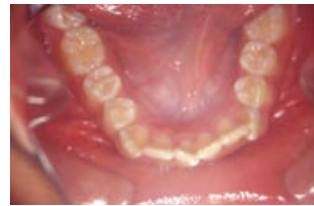

Figura 3. Trazado inicial con la cefalométria de Ricketts

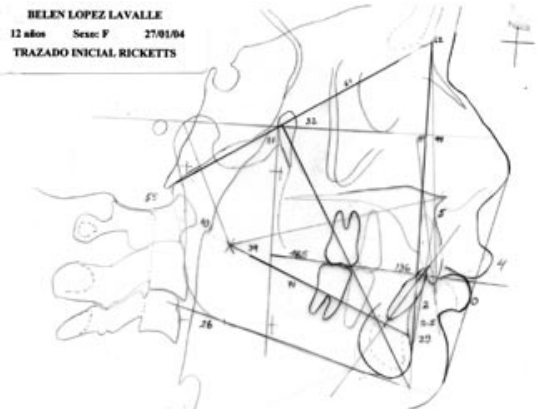

Figura 5. Objetivo visual de tratamiento VTO

Figura 4. Predicción de crecimiento para dos años
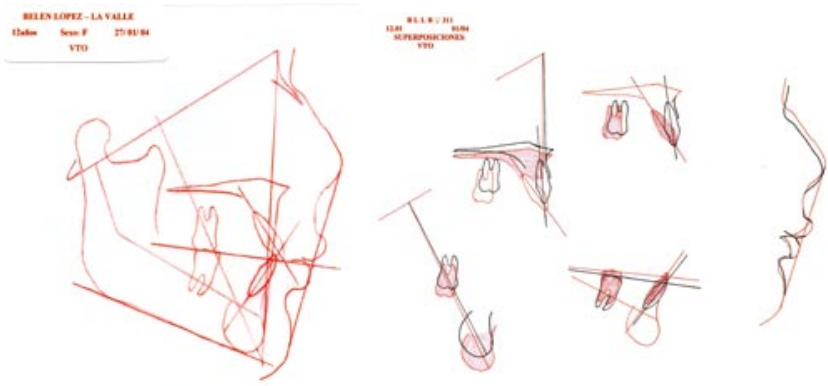

Figura 6. Mecánica inicial del tratamiento bioprogresivo con la distalización de molares, arco utilitario superior, arcos seccionales de alineamiento y arco continuo inferior
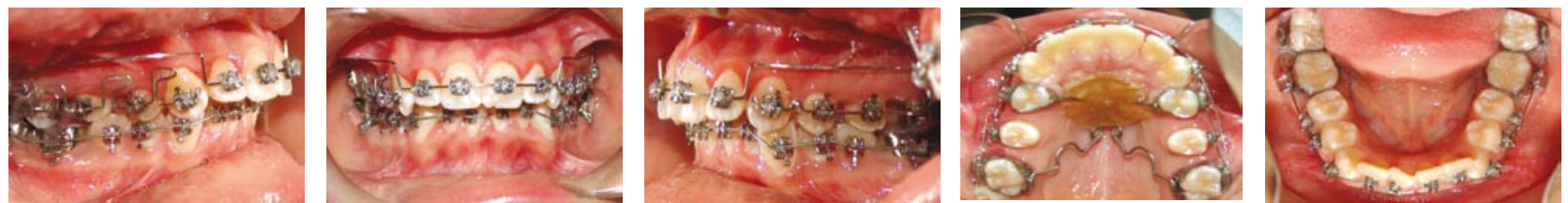

Figura 7. Mecánica de distalización de caninos y alineamiento y nivelación inferior
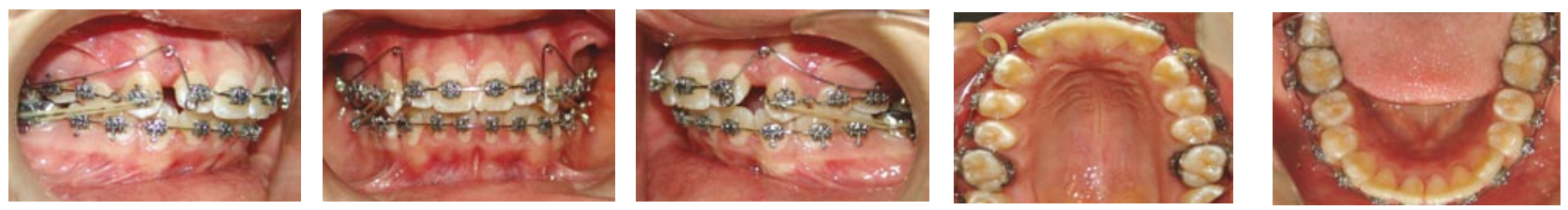
Figura 8. Elásticos 3/4 intermaxilares de interdigitación oclusal y consolidación del arco inferior con arco rectangular
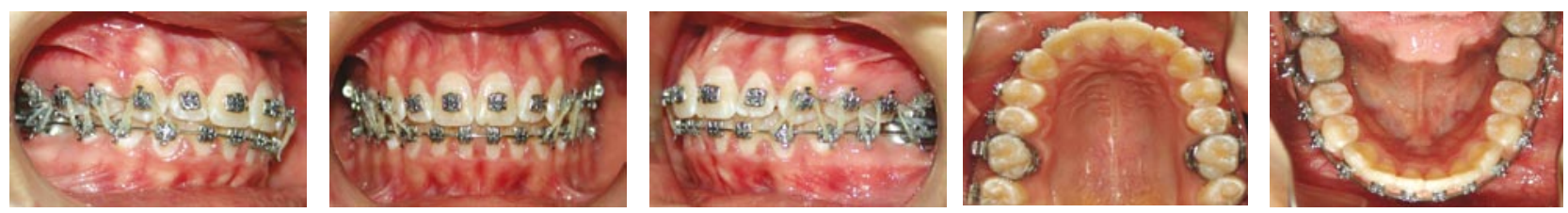

Figura 9. Evaluación del movimiento de protrusiva y guía incisiva
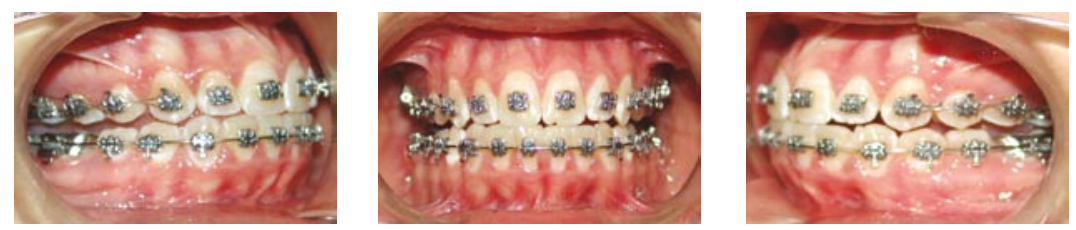

Figura 10. Evaluación de los movimientos de lateralidad derecho e izquierdo y guía canina
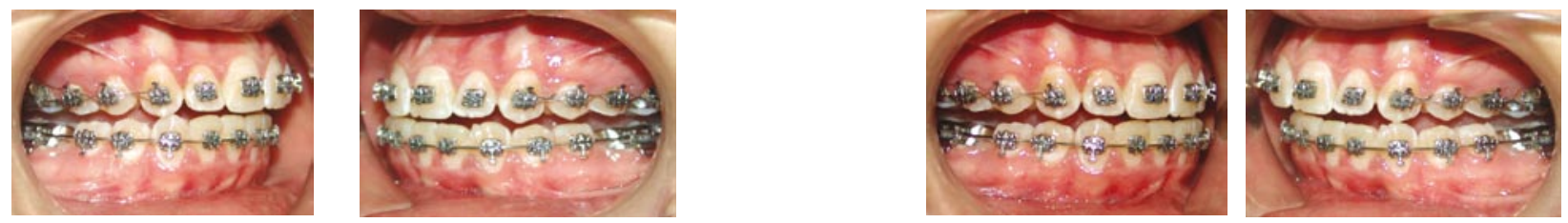

Figura 11.Fotografías intraorales y extraorales finales con la corrección de la maloclusión
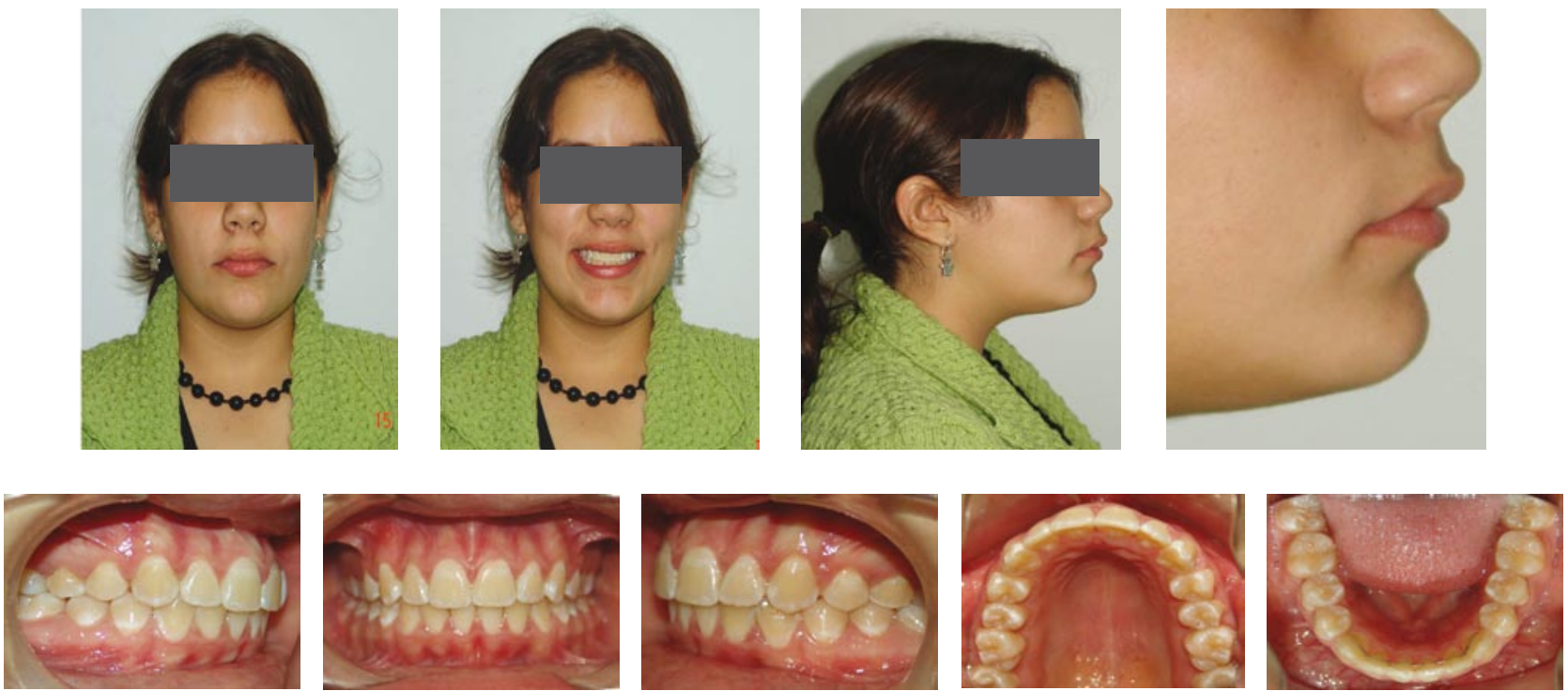
Figura 12. Radiografías cefalométrica y panorámica

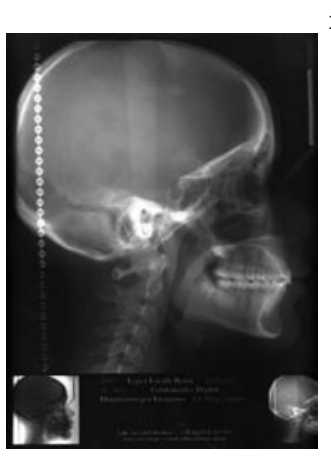

Figura 13. Cefalometría final y superposiciones cefalométricas para evaluar el tratamiento

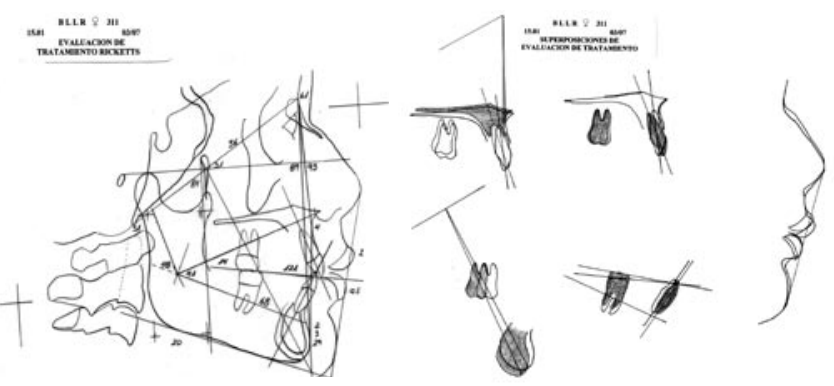

Quedó $1 \mathrm{~mm}$ desviada al lado izquierdo.

6. Mejorar la altura facial antero inferior. Mejoró en $2^{\circ}$. ENA Xi PM inicial: $39^{\circ}$ y final: $41^{\circ}$. Los valores aún son de un tercio inferior disminuido. Esto se debe probablemente a que la molar superior no se extruyó como se proyectó en el VTO. El aparato pendular no solo provocó una inhibición del crecimiento anteroposterior del la molar, también lo hizo en sentido vertical y no se extruyó probablemente por el fuerte neuromuscular de la paciente.

7. Mejorar el perfil. Mejoró, sobretodo en el labio superior, en gran parte por el efecto del cambio de inclinación de los incisivos sobre el punto A.

8. Mejorar la guía incisiva y la guía canina. Se logró darle una función bastante aceptable.

\section{Conclusiones:}

1. Una terapia sin extracciones contribuye a un mejor resultado oclusal, funcional y facial de los pacientes de Clase II División 2.

2. La corrección de la Clase II molar con el aparato pendular se produce fundamentalmente por una inhibición del crecimiento anteroposterior de la molar, además de una pequeña distalización real de la molar.

3. La aplicación de arcos utilitarios es sumamente eficiente en el manejo de la inclinación y posición de los incisivos. La terapia bioprogresiva permite un manejo adecuado de los espacios creados. Se debe destacar que el incisivo superior no se protruyó con esta terapia, tal como ocurre con la aplicación de arcos continuos.

\section{Referencias bibliográficas}

1. José A. Canut. Ortodoncia Clínica. Barcelona España: Salvat Editores. 1991:509.

2. Robertson N.R.E, Hilton R: Feature of the upper central incisors in class II division II. Angle Orthod 1965;35:5153.

3. Siriwat P.P, Jarabak J. Malocclusion and facial morphology. An epidemiologic study. Angle Orthod 1985;55:127-138.

4. Van der Linden F., Boersma H. Diagnosis and treatment planning in dentofacial orthopedics. Chicago: Quintessence.1987.

5. Hedges R.B. A cephalometric evaluation of class II division II. Angle Orthod 1958;28:191-198.

6. Fletcher G.G.T. The retroclined upper incisor. Br J Orthod 1975;2:207-216.

7. KandasamyS.,WoodsMG. Is orthodontic treatment without premolar extractions always non-extraction treatment?. Australian Dental J. 2005;50(3):146-151.
8. Kinsinger G. Molar distalization with a modified pendulum appliance-in vitro analysis of the force system and in vivo study in children and adolescents. The Angle Orthodontist. 2005;75(4):558567.

9. Hilgers J. The pendulum appliance, maintaining the gain. Clinical impressions, 1994;3(4):427-32.

10. Echarri P.A. Modified pendulum appliance for anterior anchorage control. J. Clinical Orthodontics 2003 Jul; XXXVII(7):352-359.

11. McIntyre Grant T., Millett Declan T. Lip shape and position in class II division 2 malocclusion. The Angle Orthodontist 2006;76(5):739-744.

12. Uzel Aslihan, Uzel Ilter, Toroglu MS. Two different applications of class II elastics with non-extraction segmental techniques. The Angle Orthodontist 2007;77: (2):221-225.

13. No Sangcharearn Y., Ho Christopher. Maxillary incisor angulation and its effect on molar relationships. The Angle Orthodontist 2007;77(4):694-700.

14. Uysal T., Memili B., Usumez S., Sari $Z$. Dental and alveolar arch widths in normal occlusion, class II division 1 and class II division 2. The Angle Orthodontist 2005;75(6):941-947.

Recibido : 27-04-2007

Aceptado para publicación: 25-05-2007 